\title{
Research on Multi-Hop Routing Protocol of Wireless Sensor Networks Based on Gradients
}

\author{
Chenglong $\mathrm{Hu}$ and Yongzhen $\mathrm{Li}^{*}$ \\ Department of Computer Science \& Technology YanBian University YanJi, China \\ ${ }^{*}$ Corresponding author
}

\begin{abstract}
The purposes of this paper are to lower the energy consumption in the wireless sensor network and to prolong network life cycle. Based on the maximum power a node can transmit, a multi-hop routing algorithm is proposed in this paper on the basis of gradients. We conducted a simulative experiment on the improved algorithm we proposed through MATLAB, and the results indicate that compared with LEACH algorithm and HEED algorithm, algorithm proposed in this paper is greatly improved in terms of energy consumption in the network and network life cycle.
\end{abstract}

Keywords-Wireless sensor network; broadcast radius; gradient; multi-hop transmission; network lifetime

\section{INTRODUCTION}

At present, studies of wireless sensor routing protocol have aroused many scholars' attention, yet the results are not remarkable. Except for classical routing algorithms about a decade ago, there has not been an improved routing protocol in recent years which is able to obtain support and affirmation at home and abroad [1], [2]. Therefore, there is still much to study in wireless sensor routing protocol.

Since battery energy in wireless sensor network is limited and cannot be charged [3], [4], and hardware resources and node computing abilities are all restrained, we can only seek improvements in algorithm under the circumstance that it is impossible to improve the sensor node from hardware.

Transmission distance of classical network protocol sensor nodes is limited, hindering it from transmitting at any distance. Based on this drawback, multi-hop transmission research method with gradients is proposed in this paper for the transmission distance between the nodes, so as to lower the energy consumption in the network and prolong the failure round of the first network node.

\section{RELATED WORK}

Routing protocols are divided into the following types: the plane protocol, the position-based protocol and the hierarchical protocol.

Nodes in the plane routing play the same role and each node shares the same status in the network. Flooding and Gossiping [5] are two classical algorithms in plane routing of wireless sensor network. Flooding is easy to operate, and there is no need to consume additional energy to maintain invariant topology and to achieve routing; in Gossiping, nodes are selected randomly during data transmission for relay data transmission, which avoids the information internal explosion. Yet this method takes more time for information transmission.

In routing network based on position, nodes are distinguished according to their position. Distance between the nodes is calculated according to the signal length; the longer the signal, the shorter the distance between the nodes. In some protocols, nodes are allowed to sleep when there is no operation; for instance, GEAR and GPSR [6], [7].

In hierarchical protocol, nodes are divided into clusters and the node with highest energy is selected as the cluster head. Classical algorithms of mainstream protocols in hierarchical protocol include: LEACH, LEACH-C [8], LEACH-M [9], HEED [10], PEGASIS, etc.

LEACH is a cluster protocol whose main purpose is to realize the balanced load among sensor nodes so as to prolong network life cycle. Yet there are still several problems in LEACH algorithm, which are as follows [11], [12], [13], [14], [15], [16]:

1 In LEACH, cluster head has to be selected in each round, which consumes plenty of energies. Besides, head selection doesn't take remaining energy and node position into consideration. As a result, cluster head may gather in a certain region or at the marginal zone in the area.

2 In LEACH algorithm, data are transmitted in single-hop way from member nodes to cluster head nodes, and then to the base station. When in a large-scale network, nodes away from the sink node use up their energy early due to different distances between the sink node, which is harmful to data collection. This method is unsuitable for large-scale network due to its poor expansibility.

3 Cluster head nodes transmit data to the base station directly, and therefore, these nodes consume more energies than other common nodes and lose efficiency when their energies are used up, invalidating the network too early.

LEACH-M [9] adopts the same algorithm and mechanism as those of LEACH to form clusters; at the same time, cluster head nodes record all the locations and energy information of cluster head nodes in the network.

In HEED [10] algorithm, energy consumed by the average communication in clusters is taken as the criterion to evaluate the communication cost in clusters. Key of HEED algorithm is 
that nodes decide the cluster head node through comparison of cost values between themselves and surrounding nodes.

PEGASIS algorithm organizes the nodes in the network into a chain. Its drawback is that each node must know the location information of other nodes in the network, which leads to too much expenditure and excessive routing table waiting to be maintained; when the network scale is too large, chain structure in the algorithm is too long and time delay of data transmission is relatively large, which fail to guarantee the timely data update, and chain structure cost is too large.

In a large-scale network, hierarchical routing protocol consumes less energy than plane routing protocol, and is thus more efficient. Circumstance of the research in this paper was large-scale network, so hierarchical routing protocol was selected. Algorithm realization was improved to prolong network life cycle.

\section{OuR PROPOSED METHOD}

Wireless sensor routing algorithm, which is designed in this paper based on gradients, improves the algorithm mainly in terms of cluster stage and data transmission stage.

\section{A. Cluster Stage}

\section{1) Gradient partition}

Different from the concentric circle division method of "ring-section", gradient division method which improves the algorithm was proposed based on the concentric circle division method in this paper. It recorded the number of neighboring nodes in gradient division stage, and selected intermediate nodes to forward data within the broadcast radius.

We divided the gradients in the area under the circumstance where area dimension and node number were fixed. Detailed steps of gradient division are listed as follows:

a) Initialized the gradient value of each node into 0 . Firstly, the base station delivered broadcast signal with a radius of $R$, and the message was Broadcast_message(level:1). Nodes receiving the message from base station set their gradient as 1;

b) Nodes whose gradients were 1 in the last step continued to deliver broadcast signal with a radius of $R$, and the message was Broadcast_message(level:2). Nodes who received this message and whose gradient values were 0 set their gradient as 2;

c) Circulated Step 2; divided the gradients; when each node in the network had a gradient value other than 0 , the division was finished.

d) Gradient division was over. Each node recorded number of other nodes within its broadcast radius. This figure was the neighboring node number of each node, $\mathrm{Ni}$.

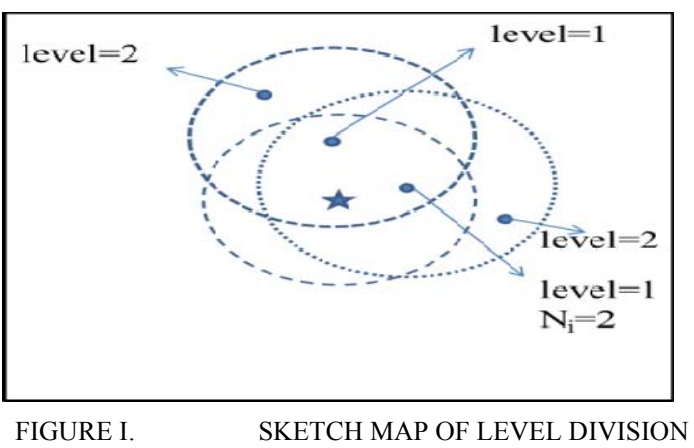

Figure 1 shows how to determine the gradient. Asterisk represents base station nodes, located in the middle of the area. In the circle which takes the base station as the center and the broadcast radius $\mathrm{R}$ as the radius, the circle is the range which the broadcast signal can reach. Divide all the regions in the whole network into different gradients according to the steps above, and each node has a gradient.

\section{2) Cluster head selection:}

In order to reduce the energy consumption, it is necessary to choose nodes with high remaining energy and suitable position as the cluster head nodes.

In classical LEACH algorithm, nodes select a random figure ranging from 0 to 1 when choosing the cluster head node, and compare it with the threshold. If the selected figure ranging from 0 to 1 is smaller than the threshold, it will be the cluster head node. This is the drawback of LEACH algorithm, and improvements on it are made in this paper. Steps of cluster head selection algorithm in this paper are as follows:

a) In each round of cluster head selection, each node selected a random figure from 0 to 1 , and compared it with the threshold $T(n)$ in formula (1). Nodes smaller than the threshold were selected as cluster head nodes;

b) Selected cluster head nodes according to the method in Step 1. Cluster head nodes delivered the broadcast message that they had become the cluster head nodes with a radius of $R$, including their identifying ID value and gradient: CH_MES(M $\left(M_{y} I D, M_{y \_} L e v e l\right)$. Other nodes which were not selected as cluster heads entered Step 3;

c) Nodes failing to be the cluster heads in Step 2 could decide which cluster to join according to the signal strength of broadcast message. They entered the cluster head nodes close to themselves, and became member nodes of the cluster head node;

d) Member nodes sent the message that they had become member nodes of the cluster head nodes to them, including identifying ID value of the cluster head and their own ID values: Join_REQ(CH_ID, $\left.M_{y \_} I D\right)$;

e) Member nodes in the cluster waited for the cluster node head to receive the information ACK, as well as the time slot TDMA the cluster head nodes distribute. Then one round of cluster head selection is over.

Threshold formula $\mathrm{T}(\mathrm{n})$ is as follows: 


$$
T(n)=\left\{\begin{array}{l}
\frac{p}{1-p(r \bmod (1 / p))}\left(\alpha * \frac{E(i)_{\text {res }}}{E_{\text {ave }}}+\beta * \frac{S(i) \cdot n}{S(i) \cdot \ln }\right), n \in G \\
0, \text { else }
\end{array}\right.
$$

$\mathrm{P}$ is the percentage of cluster head node number in the number of all the sensor nodes in the network (normally 5\%); $\alpha$ and $\beta$ are energy influence factor and density influence factor, and their sum is $1 . E_{n}$ is the remaining energy of node $n ; E_{a v g}$ is the mean remaining energy of node $n$ ' neighboring nodes; $N_{n}$ is the neighboring node number of node $n ; N_{\text {avg }}$ is the ideal neighboring node number.

Common nodes decided which cluster to join according to the signal strength, and they joined the closest cluster head node.

After member nodes joined the clusters, cluster head nodes distributed TDMA time slot towards member nodes, and broadcast the time slot of each node to member nodes in the cluster, including: TDMA(CH_ID, $\mathrm{M}_{1}: \mathrm{slot}_{1}, \mathrm{M}_{2}: \mathrm{slot}_{2}, \ldots$, $\left.\mathrm{M}_{\mathrm{n}}: \mathrm{slot}_{\mathrm{n}}\right)$. As is shown in Figure 2, the unit of wireless sensor in network routing stage is round. Each round is composed of set-up and steady state of data transmission. For a particular cluster, each round is composed of its data frames, in which each cluster member corresponds to a frame slot; and the cluster head node uses the last slot in each frame to communicate with base station.

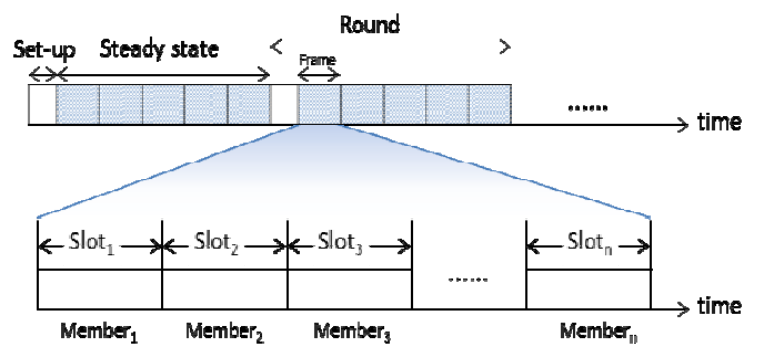

FIGURE II. SKETCH MAP OF TDMA ALLOCATION PROCESS

After the cluster head node received the message monitored by its member nodes, it integrated the information and removed the redundant message to avoid energy consumption caused by transmitting repeated message.

\section{B. Data Transmission Stage}

\section{1) Intra-cluster transmission:}

Figure 3 shows the diagram of intra-cluster transmission in data transmission. The black block is a cluster head node; the dotted circle is the assumed cluster range of this cluster head node, which is not necessarily the actual radius of this cluster head node. Cluster range of each cluster head node is not fixed, and it is only assumed here that the dotted circle is the cluster range. Each cluster transmits according to the diagram in Figure 3, after receiving member node messages, cluster head nodes integrate the data and intra-cluster transmission is over.

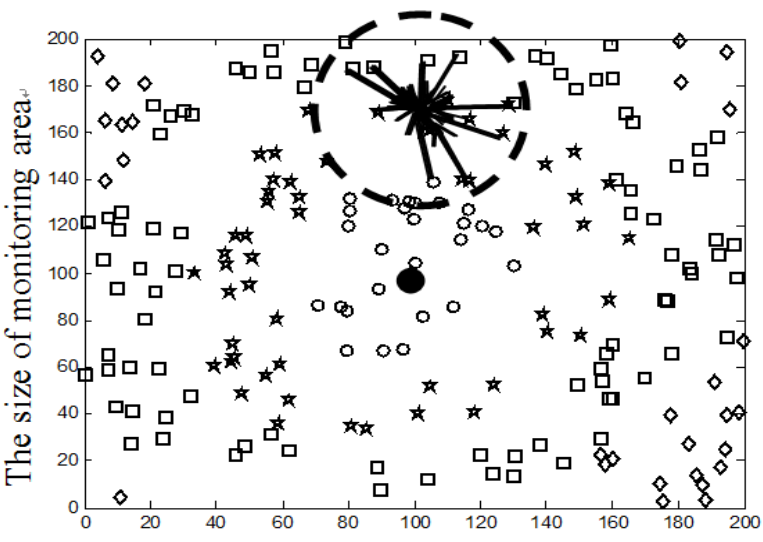

FIGURE III

SKETCH MAP OF TRANSMIT WITHIN THE CLUSTER

Figure 3 shows the diagram of intra-cluster transmission in data transmission. The black block is a cluster head node; the dotted circle is the assumed cluster range of this cluster head node, which is not necessarily the actual radius of this cluster head node. Cluster range of each cluster head node is not fixed, and it is only assumed here that the dotted circle is the cluster range. Each cluster transmits according to the diagram in Figure 3, after receiving member node messages, cluster head nodes integrate the data and intra-cluster transmission is over.

2) Transmission outside the cluster

In this paper, intermediate forwarding node was selected in the transmission outside the cluster, to forward the information from areas with large gradients into those with small ones, and eventually to the base station. Transmission algorithm outside the cluster is described as follows:

a) After intra-cluster transmission, member nodes had already sent the data to the cluster head. Now cluster head nodes needed to transmit the integrated information to sink nodes through transmission outside the cluster. Cluster head nodes whose gradients were 1 deliver the integrated information directly to sink nodes;

b) Cluster head nodes whose gradient was not 1 selected close nodes which were smaller than themselves within the broadcast radius as intermediate nodes to forward data;

c) Circulated Step 2. Selected intermediate nodes to forward the data until information was transmitted to the intermediate nodes whose gradient was 1 . Transmited the message to sink nodes directly in the end;

d) All cluster head nodes forwarded data in multi-hop according to steps above until monitored information was all transmitted to sink nodes. The transmission outside the cluster in a round of data transmission was over.

\section{SimUlation AND ANALYSIS}

\section{A. Initialization}

In this paper, we used MATLLAB to simulate the proposed algorithm and make comparisons between the proposed 
algorithm with LEACH and HEED algorithms, and verified the efficiency and rationality through study result analyses. Parameters used in the improved algorithm experiment are as follows:

TABLE I. PARAMETERS OF EXPERIMENT

\begin{tabular}{|c|c|}
\hline Parameter & Value \\
\hline Area size & $200 * 200 \mathrm{~m}$ \\
\hline Sink position & $(100 \mathrm{~m}, 100 \mathrm{~m})$ \\
\hline Number of sensor nodes & 200 \\
\hline $\begin{array}{c}\text { Ration of cluster head nodes in the total } \\
\text { number p }\end{array}$ & 0.05 \\
\hline Data package length & $4000 \mathrm{bit}$ \\
\hline Control package length & $200 \mathrm{bit}$ \\
\hline Node initial energy & $1.0 \mathrm{~J}$ \\
\hline $\begin{array}{c}\text { Energy consumption of sending and receiving } \\
\text { circuits }\end{array}$ & $50 \mathrm{~nJ} / \mathrm{bit}$ \\
\hline Free magnification & $10 \mathrm{pJ} / \mathrm{bit} . \mathrm{m}-2$ \\
\hline Multi-path magnification & $0.0013 \mathrm{pJ} / \mathrm{bit} . \mathrm{m}-2$ \\
\hline Energy consumption of data integration & $5 \mathrm{~nJ} / \mathrm{bit}$. signal- 1 \\
\hline
\end{tabular}

\section{B. Comparison of Network Survival Time}

According to the parameters in TABLE 1, as well as the certain broadcast radius $\mathrm{R}=41$, energy influence factor $\alpha=0.5$ and node density influence factor $\beta=0.5$, comparison experiment of network survival time of the algorithm in this paper, LEACH algorithm and HEED algorithm.

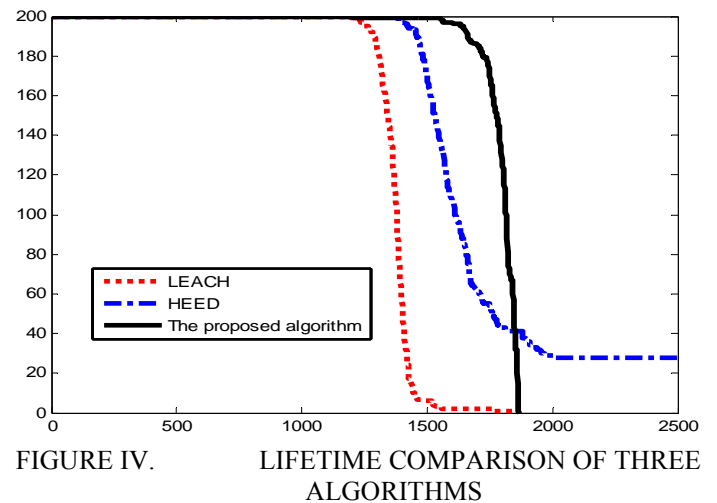

TABLE 2 shows the rounds in which the first node is invalidated of the three algorithms.

TABLE II. THE COMPARISON RESULTS OF NETWORK LIFETIME

\begin{tabular}{|c|c|}
\hline Routing Algorithm & Lifetime(round $\mathrm{n}$ ) \\
\hline LEACH & 1219 \\
\hline HEED & 1473 \\
\hline The proposed algorithm & 1711 \\
\hline
\end{tabular}

From the slopes of the curves in Figure 4, that of the algorithm in this paper is steeper than those of LEACH and HEED. With the increase of round number, the full consumption speed of node energy is slower. Advantages of the algorithm in this paper over LEACH and HEED in terms of life circle can be seen more directly from Table 2. Analyzed from the perspective of network life circle index, that of the improved algorithm raises $40.4 \%$ and $16.2 \%$ respectively, compared with LEACH and HEED. Therefore, algorithm in this paper is better than the two whether in curve slope or in detailed network life circle.

\section{Comparison of Energy Consumption in the Network}

Figure 5 is the comparison between the improved algorithm we proposed and the classic Routing Algorithm in the network energy consumption.

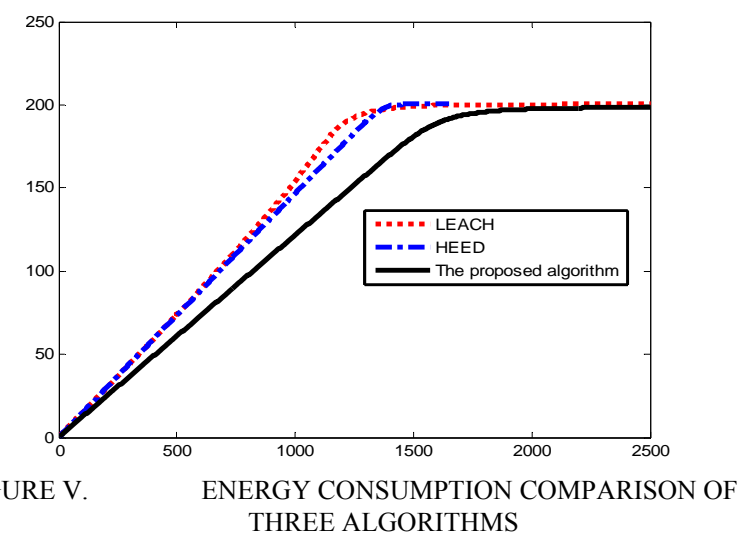

From Figure 5, curve change of this algorithm is the smoothest, that is, it has the lowest network energy consumption after the same rounds. Algorithm in this paper is more advantageous than LEACH and HEED from the round number, since with the increase of round number, cluster head nodes in LEACH have too little energy to transmit information; or cluster head nodes are distributed in areas with small or large density, which fastens the consumption of cluster head energy. When selecting cluster head nodes, this algorithm considers both the node remaining energy density among the nodes, balancing the cluster head node location distribution and cluster head node number.

\section{CONCLUSION}

In this paper, improvements are made on routing algorithm in cluster selection stage and data transmission stage, and a cluster multi-hop routing algorithm is proposed based on gradients. On the basis of simulative experiments, we analyzed property comparison of this algorithm, LEACH and HEED algorithms. In the end, we drew the conclusion that the proposed algorithm lowers the energy consumption and increases network survival time.

There are following drawbacks in the algorithm proposed in this paper and the next research plan is as follows:

1) In cluster head selection stage, that different gradients have different chances to become cluster head nodes can be considered. If we give nodes closer to the base station, that is, nodes with smaller gradients, higher possibility to become cluster heads, shorter distance to the base station leads to shorter transmission distance, which can balance the energy consumption in the network. 
2) Next step in this paper is to conduct simulative experiments closer to the real network scale. In different network scales, we study on the relationship between the parameter and network scale.

\section{REFERENCES}

[1] M. Sharma, K. Sharma, "An Energy Efficient Extended LEACH (EEE LEACH)," 2012 International Conference on Communication System and Network Technologies, Rajkot, India. pp.377 382, 2012.

[2] S. Ning, J. Yoon-su, L. Sang-ho, Energy Efficient Mechanism Using Flexible Medium Access Control Protocol for Hybrid Wireless Sensor Networks. Central South University Press and Springer-Verlag. Berlin, Heridelberg. pp.2165 2174, 2013.

[3] Shang Fengjun, Communication protocol for wireless sensor networks [M].Beijing: Publishing House of Electronics Industry.2011:1 30.

[4] Sun Limin, Li Jianzhong, Wireless sensor networks [M].Beijing: Tsinghua University Press.2010:4 21.

[5] Q. X. Xiong, C. H. Chen, "A Novel Application Semantics Based Routing Algorithm in Wireless Sensor Networks," 2014 Fourth International Conference Communication Systems and Network Technologies, Bhopal, India. pp.143 146, 2014.

[6] S. Tyagi, N. Kumar, "A Systematic Review on Clustering and Routing Techniques Based Upon Leach Protocol for Wireless Sensor Networks," Journal of Network and Computer Applications,Vol.36 No.2 pp.623 645, 2013.

[7] He Yongqiang, Gu Chunying, Wang Junpeng.,Flow divided-based uniform clustering for WSN routing algorithm [J] Application Research of Computers,2015,32(10):3075 3077.

[8] Hu Yanhua, Zhang Jianjun, Improved algorithm of cluster head multihops based on LEACH [J] Computer Engineering and Applications, 2009, 45(34):107-109.

[9] Li Yan, Zhang Xihuang, Li Yanzhong, Algorithm of cluster head multihops based on LEACH [J] Computer Engineering and Design, 2007,28(17):4158 4160.

[10] O. Younis, S. Fahmy. Heed: a Hybrid, Energy-Efficient, Distributed Clustering Approach for Ad Hoc Sensor Networks [J]. IEEE Transactions On Mobile Computing. 2004, 3(4): 366 379.

[11] A. Manjeshwar, D. Agrawal, "APTEEN: A Hybrid Protocol for Efficient Routing and Comprehensive Information Retrieval in Wireless Sensor Networks," Proceedings International on Parallel and Distributed Processing Symposium. Ft, Lauderdale, America. 2008.195 202.

[12] Lang Lili, Design and Implementation of Dijkstra Shortest Path Clustering Wireless Network Based on nRF24L01[D].BeiJing: Northeastern University,2013:37 41.

[13] J. B. Liang, J. X. Wang, "An Efficient Algorithm for Constructing Maximum Lifetime Tree for Data Gathering Without Aggregation in Wireless Sensor Networks," 2010 Proceedings IEEE INFOCOM, San Diego, America. 2010: 1 5.

[14] H. J. Liao, C. H. R. Lin, Y. C. Lin, et al, "Inntrusion Detection System: a Comprehensive Review," Journal of Network and Computer Applications, Vol.36 No.1 pp.16 24, 2013.

[15] Zhou Chong, Yu Zhenhong, Wang Nana, Research on data fusion based on clustering routing for wireless sensor network $[\mathrm{J}]$ Modern Electronics Technique,2012,35(7):43 46.

[16] S. Ning, C. Young-bok, L Sang-ho, "A Distributed Energy Efficient and Reliable Routing Protocol for Wireless Sensor Networks," Proceeding of the 14th IEEE International Conference on Computational Science and Engineering, Dalian, Liaoning. 2011: 273 278. 